\title{
On the propagation of MHD eigenmodes in a 2-D-magnetotail
}

\author{
G. Fruit and P. Louarn \\ Centre d'Etude Spatiale des Rayonnements, Université de Toulouse (UPS), Toulouse, France
}

CNRS, UMR 5187, Toulouse, France

Received: 12 July 2010 - Revised: 5 November 2010 - Accepted: 21 December 2010 - Published: 5 January 2011

\begin{abstract}
The propagation of MHD kink/sausage low frequency waves in the magnetotail with a finite normal $B_{Z}$ component is addressed. The general idea is to investigate how a finite $B_{\mathrm{Z}}$ may affect the propagation of MHD eigenmodes in the plasma sheet. The standard MHD equations are linearized and solved numerically in a modified Harris sheet. Boundary conditions are chosen such that energy flows outward of the frame box (free propagating system). An initial perturbation is set up in the pressure gradient term and the wave energy is then traced in the system. While a pure 1-D-Harris sheet constitutes an efficient wave guide for MHD eigenmodes, the introduction of a finite $B_{Z}$ in the zero-order geometry changes significantly the propagation of MHD fluctuations: the eigenmodes propagate much more slowly and carry little energy whereas a pure sound wave is excited and propagates isotropically in the system. The presence of a finite $B_{\mathrm{Z}}$ thus tends to inhibit the MHD propagation of energy along the plasma sheet. It tends rather to spread the energy throughout the magnetotail. As an application of the above study, the role of a permanent X-point structure on MHD propagation in the plasma sheet is also explored.
\end{abstract}

Keywords. Magnetospheric physics (MHD waves and instabilities)

\section{Introduction}

The Earth magnetotail has long been known to support the propagation of low frequency waves. Its stretched magnetic field structure behaves indeed as an efficient MHD wave guide (Allan and Wright, 2000). A series of theoretical studies concentrates on the coupling between fast magnetosonic waves and Alfvén waves via the phenomenon of resonant

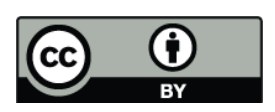

Correspondence to: G. Fruit (fruit@cesr.fr) absorption (Tamao, 1965; Chen and Hasegawa, 1974; Tirry et al., 1997; Seboldt, 1990). When a fast wave moves at a phase speed that matches the Alfvén velocity of a local field line, this line enters in resonance and energy flows from the fast wave to a very narrow Alfvén layer where it may be dissipated more easily through kinetic effects. This mechanism of mode coupling necessitates however a finite $k_{\mathrm{y}}$ component in order to excite shear Alfvén waves. On the other hand, the magnetotail also supports free propagating eigenmodes with $k_{\mathrm{y}}=0$ that do not experienced this kind of damping. These global eigenmodes constitute the basis of the linear natural response of the plasma sheet to external pressure perturbations. Theoretical investigations of the MHD propagation in a Harris current sheet show that the typical periods of oscillations are scaled by a characteristic time $\tau=a / v_{\mathrm{s}}$ where $a$ is the plasma sheet thickness and $v_{\mathrm{s}}$ is the speed of sound (Louarn et al., 2004). This parameter was later used by Fruit et al. (2004) to identify MHD eigenmodes in CLUSTER insitu magnetic measurements. In these studies the plasma sheet is modeled by a 1-D Harris sheet with no normal magnetic component $B_{\mathrm{z}}$. Although this situation may sometimes be observed, it does not correspond to a permanent feature of the magnetotail. The normal component is often non vanishing with a magnitude of the order of 2-5nT. The present paper thus proposes a theoretical analysis of MHD propagation in a 2-D-magnetotail in order to investigate how the presence of a finite $B_{\mathrm{Z}}$ may affect the existence of eigenmodes.

Few studies have indeed tackled this problem. Most of the early works restrict on pure 1-D-Harris sheet with no $B_{\mathrm{Z}}$ (Smith et al., 1997; Fruit et al., 2002). Lee and Hau (2008) investigates the case of a Harris current sheet including a guiding magnetic field $\boldsymbol{B}_{\mathrm{y}}$. Due to the twisted magnetic field in the central plasmasheet, the eigenmodes are expected to excite a local Alfvén wave when $\omega=k_{/ /} v_{\mathrm{A}}\left(z_{t}\right)$ for a particular $z_{t}$. Although it provides new insights in the MHD propagation along current sheets, they still consider a vanishing magnetic normal component. More recently Erkaev

Published by Copernicus Publications on behalf of the European Geosciences Union. 
et al. (2009a,b) analyzes the flapping motions of the magnetotail by taking into account a gradient in the normal component $B_{\mathrm{z}}$. These eigenmodes propagating perpendicular to the magnetic field (in the dawn-dusk y-direction) become unstable when $B_{Z}$ increases tailwards. They are triggered by fast plasma flows oriented earthwards. However, their model of equilibrium consider very weak $B_{\mathrm{Z}}$ component of the order of $0.02 B_{\text {lobes }} \simeq 0.6 \mathrm{nT}$. Similarly, Lysak et al. (2009) uses a low resistivity MHD code to explore the propagation of fast waves in the magnetotail that may convert into Alfvén waves to produce field aligned currents. The equilibrium of the magnetotail is first modeled by a 1-D-Harris sheet and then by a Lembège and Pellat 2-D-solution. However the magnetic normal component remains moderate, lower than $2 \mathrm{nT}$.

Here, we would like to study the influence of a moderate normal component $B_{\mathrm{Z}}$ on the propagation of perturbations polarized in the $(\mathrm{x}, \mathrm{z})$-plane with the $\mathrm{x}$-axis oriented along the tail and the z-axis pointing to the North. The y-direction is thus invariant. The disturbances are initiated by a sudden pressure burst located in the central plasma sheet. Torsional shear Alfvén waves polarized in the y-direction are not considered in this study because they are not excited by such a pressure modification. They correspond rather to variations in the current distribution and propagate parallel to the background magnetic field in a non dispersive way. On the other hand compressional modes are strongly affected by the inhomogeneity of the background plasma sheet and deserve a closer examination. Using a numerical scheme, we solve the linearized ideal MHD equations to trace the propagation of energy throughout the plasma sheet. While in the Harris sheet with $B_{\mathrm{Z}}=0$, an eigenmode structure develops clearly and carries the energy of the initial blast along the magnetic field, the introduction of a finite $B_{\mathrm{Z}}$ breaks the formation of the eigenmode structure down and the energy is essentially carried away by a pure sound wave. The nature of the MHD propagation is thus deeply modified by the presence of a transversal magnetic field.

The paper is built as the following: in Sect. 2 the equations of linear MHD are derived in 2-D magnetotail to yield a system of four partial differential equations that will be solved numerically. Section 3 explores the case of a pure Harris sheet and the numerical solution is compared to previous theoretical work which validates the numerical procedure. In Sect. 4 a small $B_{\mathrm{Z}}$ component is added in the background field and the propagation of MHD waves is traced in comparison with the 1-D-case. Finally, in Sect. 5, we investigate the problem of MHD propagation towards a permanent Xpoint embedded in the current sheet. Such a singular point may be surrounded by a high normal magnetic component that plays the role of an obstacle in the propagation of MHD eigenmodes.

\section{Theoretical model}

We derive in this section the general system of equations that governs the propagation of MHD perturbations in a 2-D magneto-plasma. The geometry uses a rectangular coordinate frame $(x, y, z)$ with the $\mathrm{x}$-axis along the magnetotail and the z-axis along the South to North direction. The y-axis is assumed to be an invariant direction in the problem. Only perturbations propagating in the (x,z)-plane are studied here. The equilibrium configuration is described by the magnetic field $\boldsymbol{B}_{0}(x, z)=B_{\mathrm{X}} \boldsymbol{e}_{\mathrm{x}}+B_{\mathrm{z}} \boldsymbol{e}_{\mathrm{z}}$ associated with the current distribution $\boldsymbol{J}_{0}=\nabla \times \boldsymbol{B}_{0} / \mu_{0}=J_{0}(x, z) \boldsymbol{e}_{\mathrm{y}}$ and the thermal pressure field $P_{0}(x, z)$ satisfy the equilibrium condition

$-\nabla P_{0}+\boldsymbol{J}_{0} \times \boldsymbol{B}_{0}=0$

The magneto-plasma is also assumed to be isothermal, thus density $\rho_{0}$ and thermal pressure $P_{0}$ are proportional through the ideal gas law. We will specify these different quantities in the next sections, investigating particular situations. Here, we consider the general frame of equations.

For future discussion, we choose the following normalization: the Earth radius $R_{\mathrm{E}} \simeq 6400 \mathrm{~km}$ scales the distances, the magnetic field is referred to its value in the lobes $B_{\text {ref }} \sim$ $25 \mathrm{nT}$, the plasma density to its value in the far neutral sheet $\rho_{\text {ref }} \sim 1 \mathrm{~cm}^{-3}$. The pressure is normalized to the magnetic pressure in the lobes $\left(P_{\text {ref }}=B_{\text {ref }}^{2} /\left(2 \mu_{0}\right)=0.25 \mathrm{nPa}\right)$ and the Alfvén speed $v_{\mathrm{A}}=B_{\mathrm{ref}} / \sqrt{\mu_{0} \rho_{\mathrm{ref}}} \simeq 560 \mathrm{~km} \mathrm{~s}^{-1}$ scales the velocities. Finally the time is normalized to the quantity $R_{\mathrm{E}} / v_{\mathrm{A}} \simeq 10 \mathrm{~s}$.

The perturbed plasma is described by a velocity field $\boldsymbol{v}$ and all other physical quantities may be decomposed according to $G=G_{0}+g$ with $G_{0}$ the quantity at equilibrium and $g$ of the order of $v$. The perturbations are assumed to be initiated by some phenomena whose origin is not necessarily described by standard MHD. Different possibilities for describing the initial perturbation may be viewed. We choose to express it as an external force $\boldsymbol{F}_{\text {ext }}$ in the righthand side of the equation of motion. For example $\boldsymbol{F}_{\text {ext }}$ could be related to a gradient in the thermal pressure. The impulsive response of a magnetic structure to an external pressure pulse is indeed particularly meaningful in the context of magnetospheric analysis. Depending on its position, such a pressure pulse could correspond to different magnetospheric processes. If it is centered on the z-axis, it can be associated to a substorm onset; if it is on the side of the sheet, it would rather model a direct solar wind perturbation. In order to browse all these possibilities the pressure pulse takes the following form

$P_{\mathrm{ext}}=P_{0} e^{-x^{2} /\left(2 L_{\mathrm{x}}^{2}\right)} e^{-\left(z-z_{0}\right)^{2} /\left(2 L_{\mathrm{z}}^{2}\right)} e^{-t / \tau}$

We assume that before $t=0$, the plasma is at rest and the external pressure term is zero. The pulse is "switched on" at $t=0$ and then decays exponentially with a time scale $\tau$. The pulse is characterized by a gaussian shape centered on $(x=0$, $z=z_{0}$ ) with dimensions $L_{\mathrm{x}}$ and $L_{\mathrm{Z}}$. Hence the external force is simply $\boldsymbol{F}=\nabla P_{\text {ext }}$. 
The linearized ideal MHD equations with a polytropic pressure law satisfied by the first order perturbed quantities $\rho, \boldsymbol{v}, p$ and $\boldsymbol{b}$ read

$$
\begin{aligned}
\frac{\partial \rho}{\partial t} & +\nabla \cdot\left(\rho_{0} \boldsymbol{v}\right)=0 \\
\rho_{0} \frac{\partial \boldsymbol{v}}{\partial t} & =-\nabla p+\boldsymbol{j} \times \boldsymbol{B}_{0}+\boldsymbol{J}_{0} \times \boldsymbol{b}+\boldsymbol{F}_{\mathrm{ext}} \\
\boldsymbol{j} & =\frac{\nabla \times \boldsymbol{b}}{\mu_{0}} \\
\frac{\partial \boldsymbol{b}}{\partial t} & =\nabla \times\left(\boldsymbol{v} \times \boldsymbol{B}_{0}\right) \\
\left(\frac{\partial}{\partial t}+\boldsymbol{v} \cdot \nabla\right) \frac{P}{\rho^{\gamma}} & =0
\end{aligned}
$$

After combining Eqs. (3) and (7) and introducing the sound velocity $c_{\mathrm{s}}=\sqrt{\gamma P_{0} / \rho_{0}}$ we get

$\frac{\partial p}{\partial t}=-\rho_{0} c_{\mathrm{s}}^{2} \nabla \cdot \boldsymbol{v}-\boldsymbol{v} \cdot \nabla P_{0}$

or by integrating in time

$p=p^{*}-\xi \cdot \nabla P_{0}$

with $\boldsymbol{\xi}$ the displacement given by $\boldsymbol{v}=\partial \boldsymbol{\xi} / \partial t$. $p^{*}$ is the classical thermal pressure fluctuations produced by the gradient in the velocity field. The introduction of Eq. (9) in Eq. (4) yields the term $\nabla\left(\xi \cdot \nabla P_{0}\right.$ which may be transformed using the equilibrium condition (1) and after some algebra

$$
\begin{aligned}
\nabla\left(\boldsymbol{\xi} \cdot \nabla P_{0}\right) & =-\boldsymbol{J}_{0} \times \boldsymbol{b}+\left(\boldsymbol{B}_{0} \times \boldsymbol{\xi}\right) \times\left(\nabla \times \boldsymbol{J}_{0}\right) \\
& +\left[\left(\boldsymbol{B}_{0} \times \boldsymbol{\xi}\right) \cdot \nabla\right] \boldsymbol{J}_{0}+\left(\boldsymbol{J}_{0} \cdot \nabla\right)\left(\boldsymbol{B}_{0} \times \boldsymbol{\xi}\right)
\end{aligned}
$$

The first term in the right-hand side of Eq. (10) cancels its equivalent in Eq. (4). The last two terms vanish because both $\boldsymbol{J}_{0}$ and $\boldsymbol{\xi} \times \boldsymbol{B}_{0}$ are oriented along the y-axis and the problem is independent of $y$. Thus the equation of motion (4) simplifies into

$\rho_{0} \frac{\partial \boldsymbol{v}}{\partial t}=-\nabla p^{*}-\left(\boldsymbol{B}_{0} \times \boldsymbol{\xi}\right) \times \frac{\Delta \boldsymbol{B}_{0}}{\mu_{0}}+\frac{\nabla \times \boldsymbol{b}}{\mu_{0}} \times \boldsymbol{B}_{0}+\boldsymbol{F}_{\mathrm{ext}}$

We can still simplify the system of governing equations by reducing the number of variables by one. The 2-D magnetic field can indeed be expressed in terms of a unique flux function $\psi$ through the relation $\boldsymbol{b}=\nabla \times\left(\psi \boldsymbol{e}_{\mathrm{y}}\right)$. Equations (6) and (11) can be rewritten as

$$
\begin{aligned}
\frac{\partial \psi}{\partial t} & =\left\|\boldsymbol{v} \times \boldsymbol{B}_{0}\right\| \Leftrightarrow \psi \boldsymbol{e}_{\mathrm{y}}=\boldsymbol{\xi} \times \boldsymbol{B}_{0} \\
\rho_{0} \frac{\partial \boldsymbol{v}}{\partial t} & =-\nabla p^{*}+\left(\psi \boldsymbol{e}_{\mathrm{y}}\right) \times \frac{\Delta \boldsymbol{B}_{0}}{\mu_{0}} \\
& -\frac{\Delta \psi}{\mu_{0}}\left(\boldsymbol{e}_{\mathrm{y}} \times \boldsymbol{B}_{0}\right)+\boldsymbol{F}_{\mathrm{ext}}
\end{aligned}
$$

In summary the problem is described by the four unknown quantities $v_{x}, v_{z}, p^{*}$ (named $p$ thereafter) and $\psi$. They satisfy the following coupled partial differential equations

$\frac{\partial v_{x}}{\partial t}=-\frac{1}{\rho_{0}} \frac{\partial p}{\partial x}+\frac{\Delta B_{\mathrm{z}}}{\mu_{0} \rho_{0}} \psi-\frac{B_{\mathrm{z}}}{\mu_{0}} \rho_{0} \Delta \psi+F_{x}$

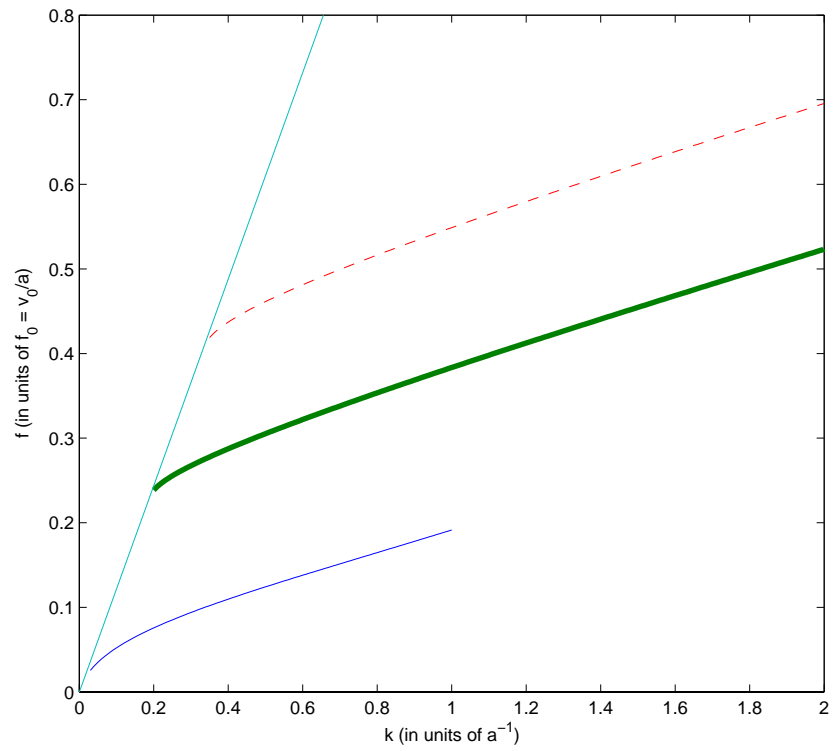

Fig. 1. Dispersion curve for the evanescent MHD eigenmodes of a Harris sheet. Frequency in units of $v_{\mathrm{A}} / a$ is plotted against the wave number in units of $a^{-1}$. Thin and dashed lines are the first and second kink mode. Thick line is the first sausage mode. All the dispersion curves stem from the $f=k v_{\mathrm{A}}$, lobes $/(2 \pi)$ line with $v_{\mathrm{A}, \text { lobes }}=7.4 v_{\mathrm{A}}$ is the Alfvén velocity in the lobes.

$$
\begin{aligned}
\frac{\partial v_{z}}{\partial t} & =-\frac{1}{\rho_{0}} \frac{\partial p}{\partial z}-\frac{\Delta B_{\mathrm{x}}}{\mu_{0} \rho_{0}} \psi+\frac{B_{\mathrm{x}}}{\mu_{0}} \rho_{0} \Delta \psi+F_{y} \\
\frac{\partial p}{\partial t} & =-\rho_{0} c_{s}^{2}\left(\frac{\partial v_{x}}{\partial x}+\frac{\partial v_{z}}{\partial z}\right) \\
\frac{\partial \psi}{\partial t} & =B_{\mathrm{z}} v_{x}-B_{\mathrm{x}} v_{z}
\end{aligned}
$$

We solve this system numerically using a finite difference scheme with $\Delta x=0.2$ and $\Delta z=0.1$. The frame box extends from $x=-20$ to $x=20$ and $z=-10$ to $z=10$. As we are primarily interested in perturbations confined within the plasma sheet (say $|z|<1 R_{\mathrm{E}}$ ), we choose for simplicity Dirichlet conditions on the boundaries of the frame box and to avoid the problem of spurious reflections we add an artificial dissipative mechanism that kills the perturbations drastically for $|z|>z_{\mathrm{m}}$ where $z_{\mathrm{m}}$ is adjusted according to the equilibrium model considered. This artificial term is of the form $-\alpha v_{x, z}$ in Eqs. (14)-(15) with $\alpha=0$ for $|z|<z_{\mathrm{m}}$ and $\alpha=\left|z-z_{\mathrm{m}}\right|$ for $z>\left|z_{\mathrm{m}}\right|$. If $z_{\mathrm{m}}$ is chosen to be a few $R_{\mathrm{E}}$ the effect of this diffusive mechanism plays a minor role in the propagation of energy inside the current sheet. Moreover, we check that changing the precise position of $z_{\mathrm{m}}$ does not affect significatively the numerical solution. 


\section{Propagation in a 1-D-Harris sheet}

We begin our investigations with a pure 1-D-Harris sheet with the following equilibrium quantities

$$
\begin{aligned}
& B_{\mathrm{X}}=-\tanh (z / a) \\
& B_{\mathrm{Z}}=0 \\
& \rho_{0}=r+\frac{1}{\cosh ^{2}(z / a)}
\end{aligned}
$$

where $a$ is the typical thickness of the plasmasheet ( $a=$ $\left.1 \quad R_{\mathrm{E}}\right)$ and $r$ is the density in the lobes $(r=1 / 50 \sim$ $\left.0.02 \mathrm{~cm}^{-3}\right)$. Sound velocity is chosen to be equal to $c_{\mathrm{S}}=$ $\sqrt{\gamma / 2}$. This standard solution of the kinetic equations (Harris, 62) is a good model of the far tail where the magnetic field is almost aligned along the x-direction. Obviously closer to the Earth the transversal component $B_{\mathrm{Z}}$ cannot be ignored any longer and this model becomes unrealistic.

With this equilibrium configuration, the system of MHD equations may transform into a single ordinary differential equation (say on $v_{z}$ ) and the initial value problem can be solved using standard analytical techniques such as Laplace transform and Bromwich integrals in the complex $\omega$-plane (Sedlacek, 1971; Fruit et al., 2002). Fruit et al. (2002) concentrates on evanescent modes in the lobes, they compute the dispersion relation for these MHD eigenmodes that can propagate along the magnetotail waveguide. Here we shall solve the problem completely numerically and compare the solution to this previous theoretical work. This may be also regarded as a test for the numerical procedure. To that end, Fig. 1 shows the dispersion diagram for the MHD eigenmodes. Thin solid line and dashed lines correspond to symmetric kink modes and the thick line to the first antisymmetric sausage mode. To visualize more clearly the dynamics of the plasma sheet, Fig. 2 shows the displaced magnetic field lines and the thermal pressure fluctuations (in normalized units) produced by each of these eigenmodes. Black/white regions correspond to high/low thermal pressure. For the sausage mode, the thermal pressure fluctuations are symmetric with respect to the $z=0$-plane; an increase in $p_{\text {th }}$ leads the field lines to move further apart in an antisymmetric way. For the kink mode, all the field lines are moving roughly parallel to each other and the thermal pressure fluctuations are antisymmetric with respect to the $z=0$-plane. All the dispersion curves are located under the $\omega=k v_{\mathrm{A} \text {, lobes }}$ line where $v_{\mathrm{A} \text {,lobes }}$ is the Alfvén velocity in the lobes. Above that line modes radiate energy outwards and are not confined within the current sheets. These modes were not considered in the work of Fruit et al. (2002).

Figures 3 and 4 represent the normal velocity $v_{z}$ and the thermal pressure $p$ as contour plots in the $\mathrm{x}, \mathrm{z}$-plane at different times. The left column corresponds to times $0.2,0.4$, 0.6, 0.8 and 1 and illustrates the transient regime immediately following the pressure burst. The right column shows the further development of the perturbation at times 4,8 ,
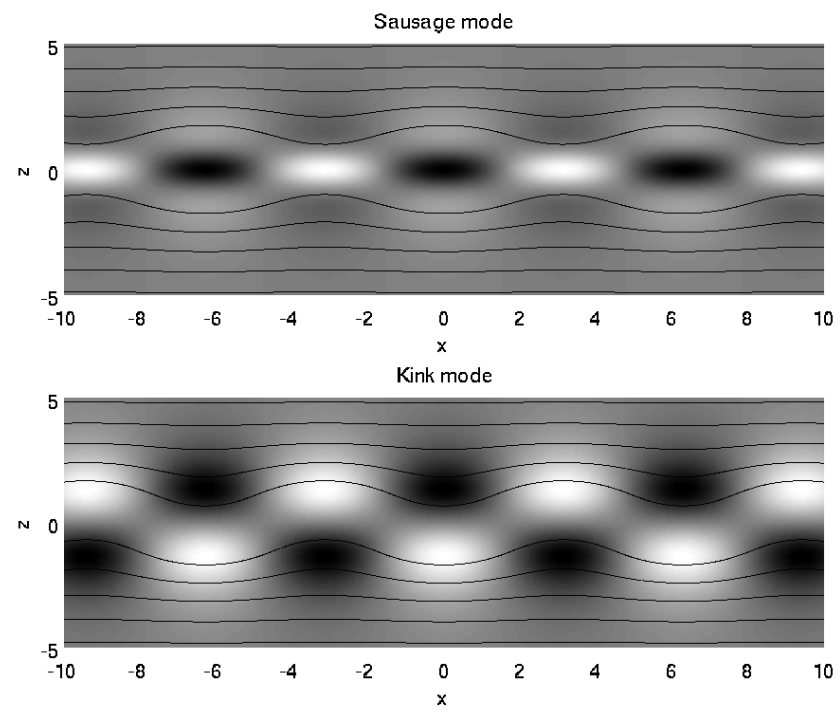

Fig. 2. Sketch of the dynamics of the plasma sheet in a sausage mode (top) and in a kink mode (bottom) configuration. Perturbed magnetic field lines are drawn together with contour plot of thermal pressure fluctuations. Black spots correspond to a higher pressure whereas white spots denotes lower thermal pressure. As the total pressure is roughly conserved, a decrease in $p_{\text {th }}$ is accompanied by an increase in the magnetic pressure and field lines move closer to each other, or vice-versa.

12, 16 and 20. Remember that times are scaled according to the Alfvén time $t_{\mathrm{A}}=R_{\mathrm{E}} / v_{\mathrm{A}} \simeq 10 \mathrm{~s}$. For this run the initial pressure pulse is specified by $z_{0}=a, L_{\mathrm{X}}=a, L_{\mathrm{Z}}=a / 2$ and $\tau=0.1$, a value of $z_{\mathrm{m}}=15$ has also been chosen and the grid extends to $z=20$, hence perturbations entering the band $15<|z|<20$ are strongly damped and do not reflect on the boundary. The tail is displayed only in the high gradient zone for $|z|<a$. By choosing an initial pulse not centered on the equatorial plane, we are ensured that both kink and sausage eigenmodes are excited. In that way all the possibilities are explored in order to test the numerical code. If the initial pulse was put on the $x$-axis, only antisymmetric sausage modes would be excited but they would propagate the same way as shown on Fig. 3 and 4. It can be seen on Fig. 3 that a fast magnetosonic wave propagates northward with the leading edge traveling at the Alfvén velocity in the lobes $v_{\text {lobes }}=7.5 v_{\mathrm{A}}$. However, due to the non homogeneous Alfvén speed profile, the propagating front is not isotropic as it would be expected from a fast wave. The wave does not propagate in the negative $\mathrm{z}$-direction towards the neutral sheet where the Alfvén velocity vanishes. It is interesting to note that this fast wave has practically no effect on thermal pressure for $z>1$ because in the edge of the plasma sheet the magnetic field becomes dominant with a low $\beta$. 

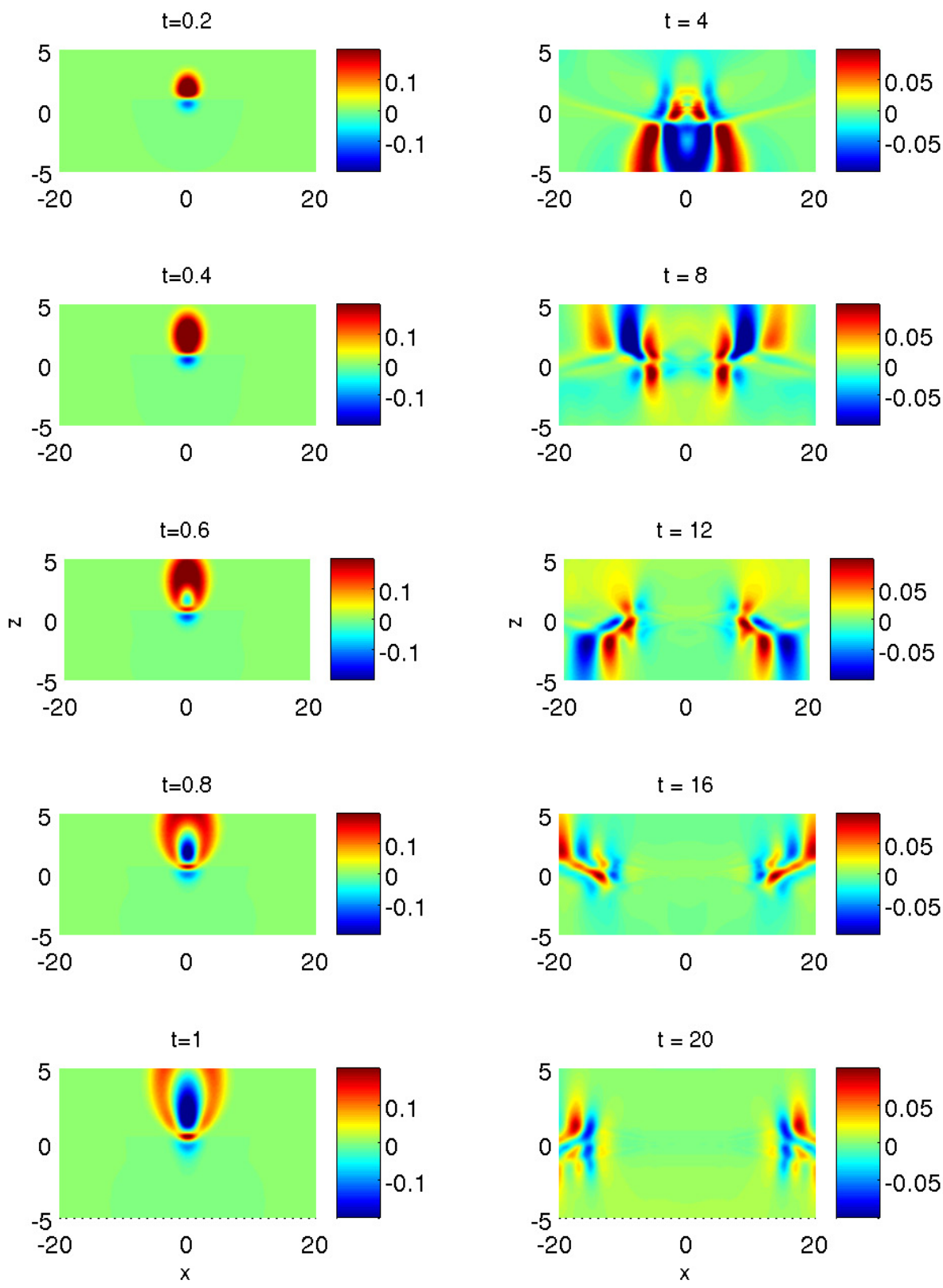

Fig. 3. Normal velocity $v_{z}(x, z, t)$ in the $\mathrm{x}$-z-plane for different values of time showed above each graph. The left column describes the first instants following the pressure burst: a fast wave is clearly expanding in the northern lobe. The right column shows the further evolution of the propagation for longer times: the eigenmode structure localized within the current sheet propagates along the $\mathrm{x}$-axis.

In the second part of the propagation, the development of the MHD eigenmodes can be observed from $t=4$ to $t=20$. A mixture of kink and sausage modes analogous to Fig. 2 is seen to propagate along the magnetic field lines. To make it clearer Fig. 5 represents the temporal oscillations of the normal velocity $v_{z}$ at $x=10$ and $z=1$. In order to compare these oscillations with the theoretical dispersion diagram the signal has been split in a symmetric part (the solid line) $\left(v_{z}(z)+v_{z}(-z)\right) / 2$ and in a antisymmetric part (the dashed line) $\left(v_{z}(z)-v_{z}(-z)\right) / 2$. The power spectrum of these two signal is added below. The structure in eigenfrequencies is obvious. The eigenfrequencies of the two main kink modes are at $0.16 f_{0}$ and $0.45 f_{0}$ with $f_{0}=v_{\mathrm{A}} / a=0.1 \mathrm{~Hz}$. This corresponds on the dispersion curve (Fig. 1) to wavenumbers $0.5-0.8$ or to wavelength ranging from 8 to $12 R_{\mathrm{E}}$. The first sausage mode has a frequency of $0.25 f_{0}$ which corresponds to the left edge of the dispersion curve (thick line). This shows a good agreement between the full numerical solution of the initial value problem and the theoretical analysis of the MHD eigenmodes in a Harris sheet. The numerical model is thus validated. 

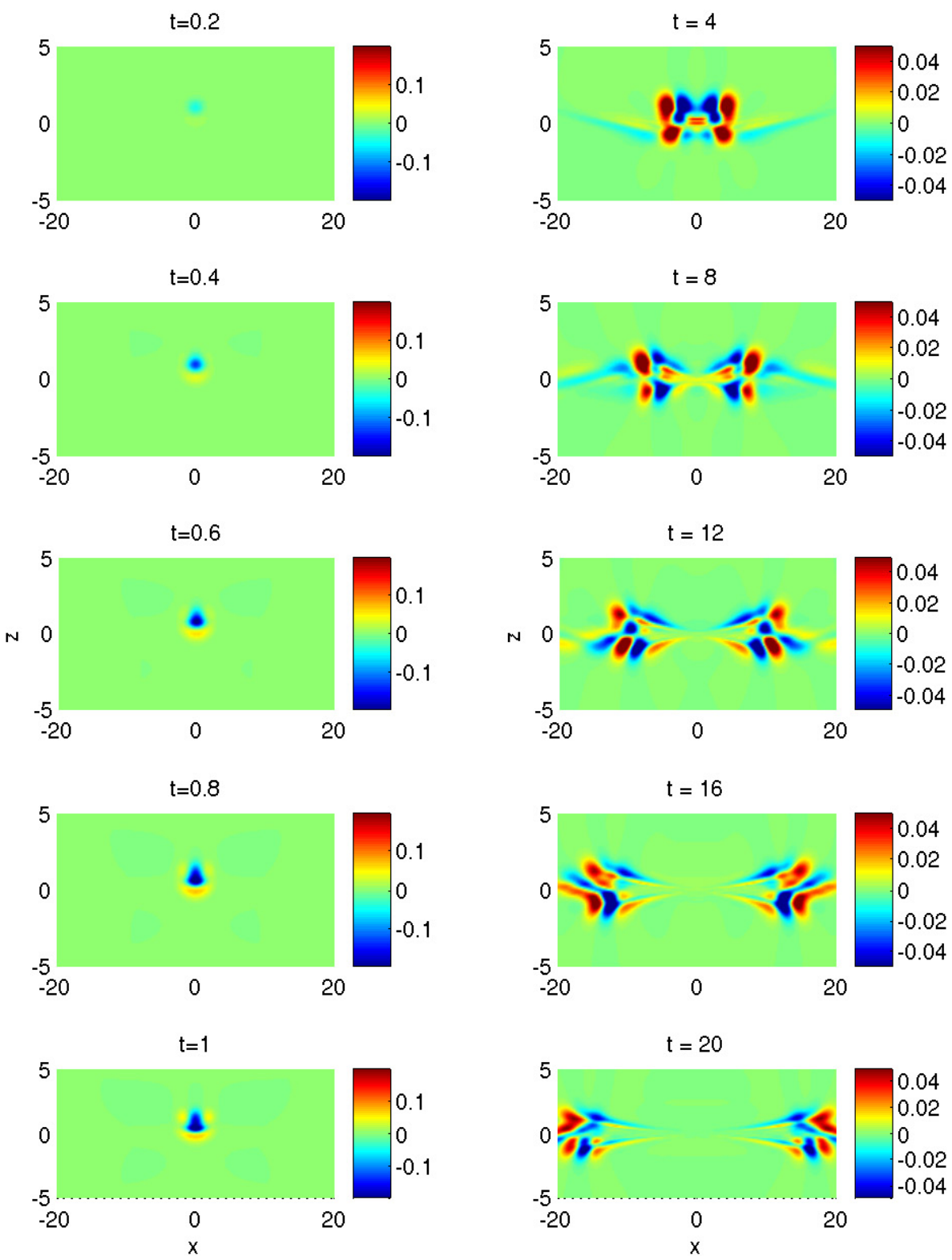

Fig. 4. Thermal pressure $p(x, z, t)$ in the x-z-plane for various times. As in Fig. 3 the left column displays the first Alfvén time and the right column corresponds to the further evolution of the perturbations. Here also the eigenmode structure (a mixing of kink and sausage mode) propagates along the static magnetic field, but the initial fast wave does not have a strong effect on the thermal pressure.

As the theoretical work of Fruit et al. (2002) concentrates on MHD eigenmodes only and does not include the initial fast magnetosonic wave, it is interesting here to complete the analysis by evaluating how much power the transient wave carries away and how much energy is left to the confined eigenmodes. To that end we delimitate a rectangular frame centered at the origin with dimensions $40 \times 10$ and we evaluate the Poynting flux across this frame. Poynting vector has two components $R_{x}=e_{t} v_{x}$ and $R_{z}=e_{t} v_{z}$ where

$e_{t}=\frac{1}{2} \rho v^{2}+\frac{B^{2}}{2 \mu_{0}}+\frac{p_{\mathrm{th}}}{\gamma-1}$ is the energy density. To distinguish between the two contributions, we make the following crude assumption. The transient wave propagates mainly along the $\mathrm{z}$-direction whereas the eigenmodes are mostly guided along the $\mathrm{x}$-direction. Accordingly the power transported by each wave may be approximated by

$$
\begin{aligned}
P_{\text {trans }}(t) & =\int_{-20}^{20} R_{z}(x, z= \pm 5) d x \\
P_{\mathrm{eg}}(t) & =\int_{-5}^{5} R_{x}(x= \pm 20, z) d z
\end{aligned}
$$



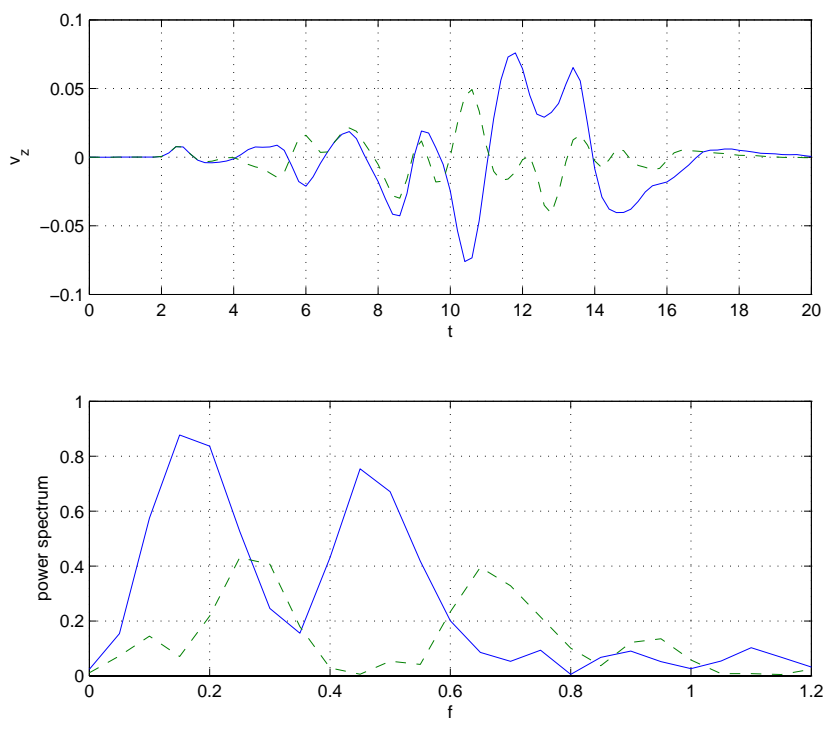

Fig. 5. Top panel: normal velocity of the plasma sheet recorded at $x=$ and $z=$ along time. In order to separate the kink and sausage mode, the half sum (solid line) and the half difference (dashed line) of the velocity field is considered as explain in the text. Bottom panel: power spectrum of the above fluctuations in $v_{z}$, two harmonics compose the kink and sausage fluctuations.

The reality is probably more complex but this order of magnitude evaluation would be sufficient to get a good insight on how the energy flows in the plasma sheet. Figure 5 shows the two powers $P_{\text {trans }}$ (in solid line) and $P_{\text {eg }}$ (in dashed line) as functions of time. The maximum power transported by each mode is of the same order of magnitude. This means that only half of the initial energy contained in the pressure pulse remains within the plasmasheet where it may be dissipated through non linear processes. The other half propagates away from the neutral sheet towards the lobes and is likely to have little effect on plasmasheet dynamics. Converted to real units, the power associated with the MHD eigenmode is of the order of $0.08 B_{\text {ref }}^{2} /\left(2 \mu_{0}\right) \times v_{\text {ref }} \simeq 10^{-5} \mathrm{~W}$ for a pressure burst of $0.25 \mathrm{nPa}$.

\section{Propagation in a 2-D modified Harris sheet}

The Harris sheet model becomes unsatisfactory when it is applied to the near Earth region of the magnetotail where a non negligible $B_{\mathrm{Z}}$ component must be taken into account. Topologically speaking the presence of a $B_{\mathrm{Z}}$ modifies quite deeply the geometry of the plasma sheet. In particular it connects magnetically the two lobes and breaks the symmetry along the z-axis. It is then interesting to investigate how low frequency MHD fluctuations behave in this new configuration. In order to satisfy $\nabla \cdot \boldsymbol{B}=0$, the simplest improvement to the Harris sheet that may be thought of is to add a constant $B_{\mathrm{Z}}$ to the equilibrium (18). In order to satisfy the equilibrium Eq. (1) the thermal pressure must vary along the x-direction

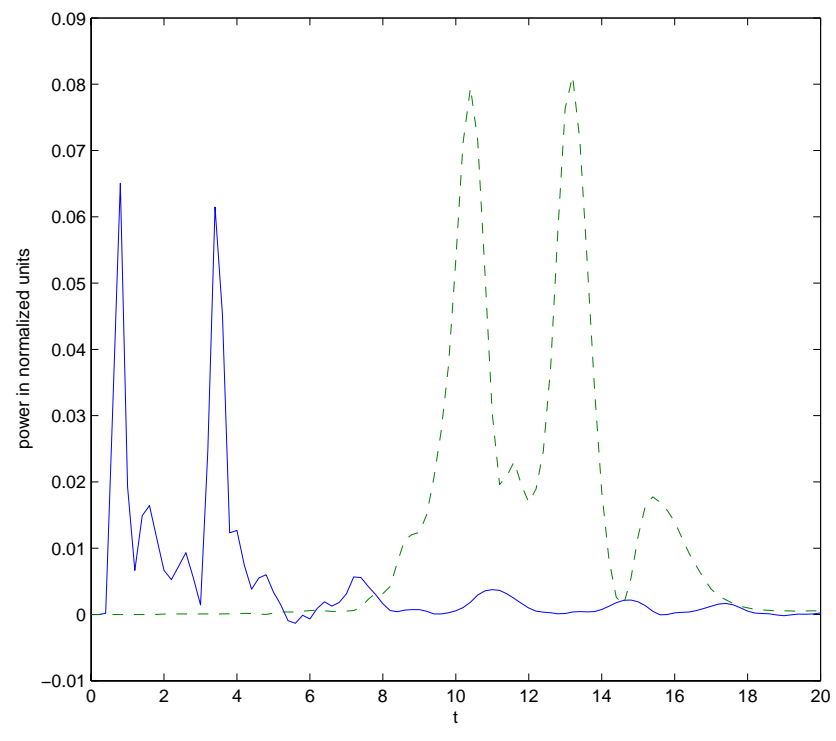

Fig. 6. A comparison between the power carried away by the initial fast wave $P_{\text {trans }}$ (solid line) and the power $P_{\text {eg }}$ transported by the eigenmodes along the plasma sheet (dashed line) in the case of a pure 1-D-Harris sheet $\left(B_{\mathrm{Z}}=0\right)$. The initial energy is roughly equipartitioned between the two propagating waves with a slight advantage for the eigenmode structure.

as well as in the z-direction. If the magnetotail is still considered as isothermal, the plasma density now takes the form

$\rho(x, z)=1-(1-r)\left(2 B_{\mathrm{Z}} x+\tanh ^{2}(z / a)\right)$

The main inconvenience of such a configuration is that it is no longer an exact solution of the Vlasov equation and it describes only approximately the real magnetic field in the tail. An alternative would be to consider a Lembège-Pellat equilibrium (Lembege and Pellat, 1982) but in that case, the transversal component $B_{\mathrm{Z}}$ remains small compared to the magnetic field in the lobes (typically $B_{\mathrm{Z}} \simeq 0.01 B_{\text {ref }}$ ). Measurements by CLUSTER or THEMIS have however proved that $B_{\mathrm{Z}}$ can be much larger reaching 5 to $10 \mathrm{nT}$ or in normal-

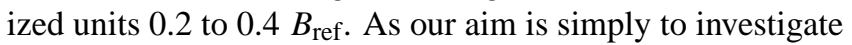
the role of a transversal component $B_{\mathrm{Z}}$ on the propagation of MHD eigenmodes, it seems easier in a first attempt to consider a constant $B_{\mathrm{Z}}$ coupled with a Harris sheet.

Figure 7 displays the thermal pressure in $(\mathrm{x}, \mathrm{z})$-plane at times $t=2$ (left column) and $t=20$ (right column) and using the same initial perturbation centered at $z_{0}=a$ (see Eq. 2). Three values of $B_{\mathrm{Z}}$ are chosen: from top to bottom $B_{\mathrm{Z}}=0,0.01,0.1 B_{\text {ref }}$. The equilibrium field lines have been added in the background. It can be clearly observed that the addition of even a small $B_{\mathrm{Z}}$ component changes radically the propagation of MHD perturbations. First of all, during the first Alfvén times $(t \lesssim 2)$, the pressure burst yields to the formation of a front propagating almost isotropically in the plasmasheet whereas no pressure wave was observed in the pure Harris sheet. The speed of this traveling front decreases 

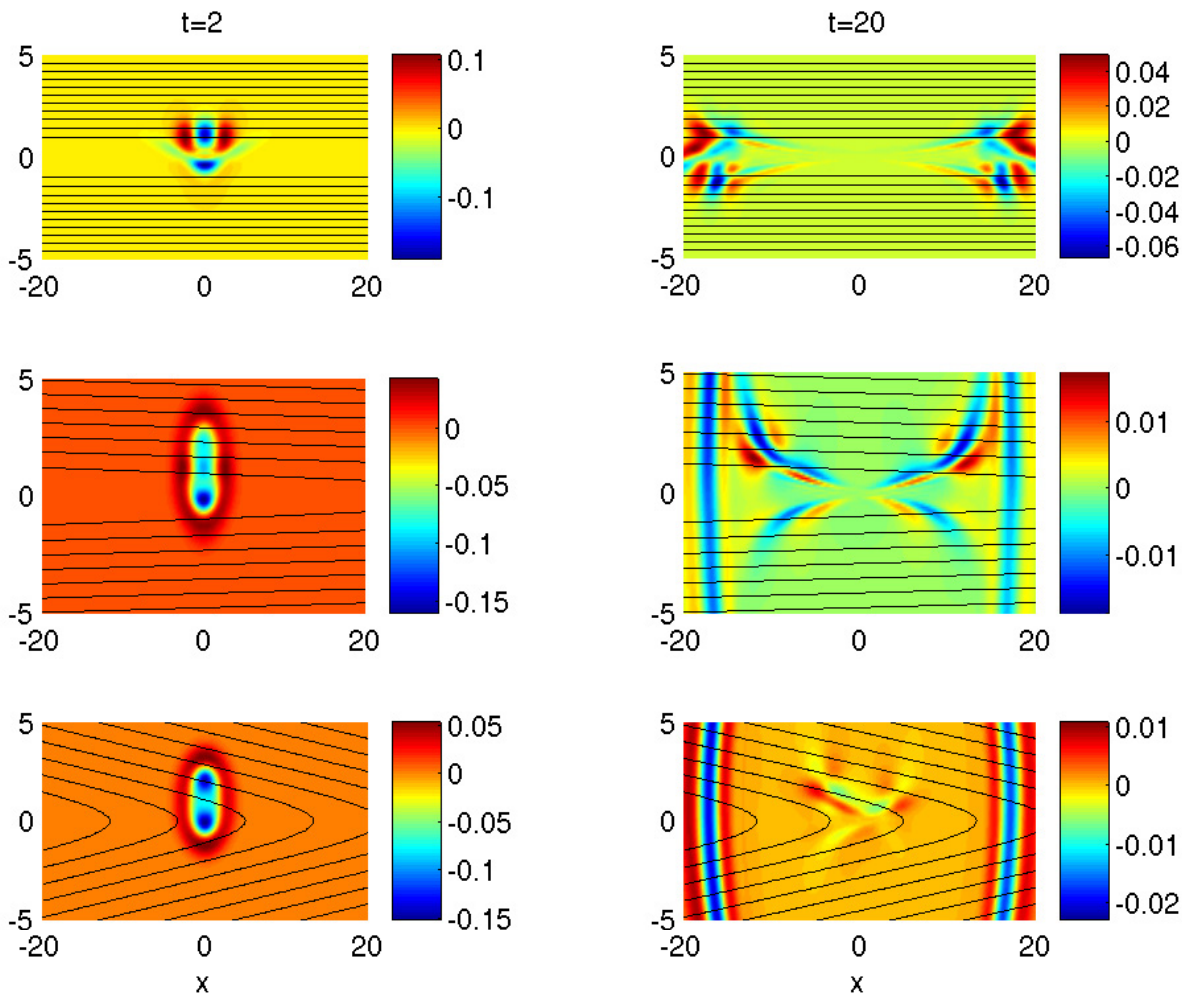

Fig. 7. Influence of a small $B_{\mathrm{Z}}$ component on the propagation of the eigenmodes of the plasma sheet. Thermal pressure $p(x, z, t)$ is plotted in the X-z-plane for two values of time: $t=2$ on the left column and $t=20$ on the right column. From top to bottom the normal components is $B_{\mathrm{Z}}=00.010 .1 B_{\text {ref. }}$. Background field lines are also displayed. While an eigenmode structure develops very quickly in the 1-D case it takes longer to develop with the presence of a $B_{\mathrm{Z}}$. The higher the normal field is the slower is the propagation of the eigenmodes.
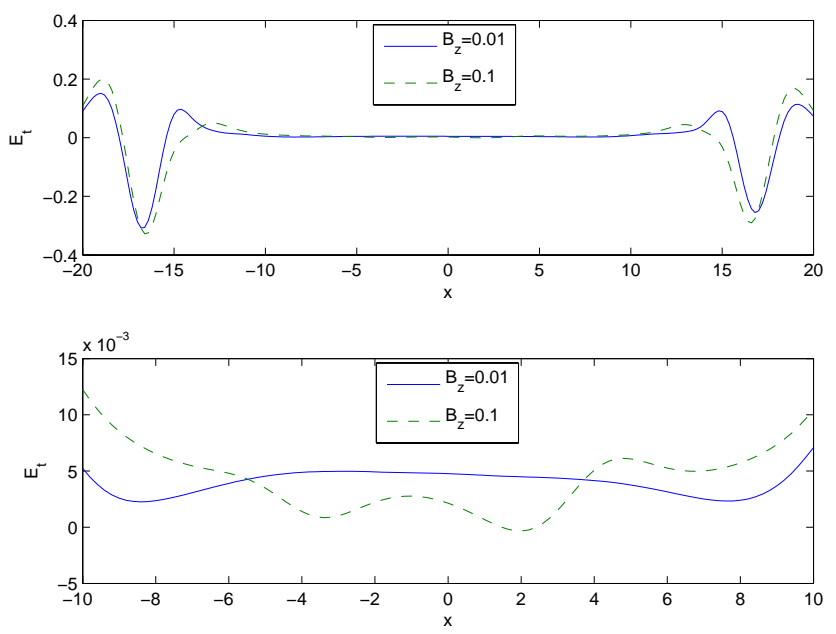

Fig. 8. The total energy density integrated in the z-direction is plotted as function of $x$ at $t=20$ in the upper panel. The solid line corresponds to $B_{\mathrm{Z}}=0.01$ and the dashed line to $B_{\mathrm{Z}}=0.1$. The fluctuations of energetic importance are located at $x= \pm 18$ and correspond to a simple sound wave. The MHD eigenmodes situated between $x= \pm 10$ contain relatively little energy. strongly with $B_{\mathrm{Z}}$. It is close to the Alfvén speed in the lobes when $B_{\mathrm{Z}}=0$ and drops to the speed of sound $\left(v_{\mathrm{s}} \simeq 0.9 v_{\text {ref }}\right)$ when $B_{\mathrm{Z}}>0.1 B_{\text {ref. }}$.

Further in time, at $t=20$, the MHD eigenmodes structure is fully developed but its extension along the magnetotail axis varies greatly with $B_{\mathrm{Z}}$. While the eigenmodes are efficiently guided along the Harris sheet, the propagation of the pressure perturbations appears much slower in the presence of a small $B_{\mathrm{z}}$. With $B_{\mathrm{z}}=0.1 B_{\text {ref }} \simeq 2.5 \mathrm{nT}$ the propagation velocity along the $\mathrm{x}$-axis has been divided roughly by 4 . This result shows that a 2-D current sheet with a relatively strong $B_{Z}$ component loses its efficiency in supporting low frequency eigenmodes over large distances.

The second main difference between the three geometries is the relative amplitude of the eigenmodes compared to the initial wave front. It can be seen on the lower right panel of Fig. 7 that most of the pressure fluctuations are carried away by the sound wave propagating independently with the magnetic configuration, leaving little energy to the eigenmodes themselves. To make the comparison clearer, we compute the total energy stored at each time $t$ in a vertical slice located at a given $x$ (in other words, the total energy density is integrated in the $\mathrm{z}$-direction for each $(x, t))$. The upper panel of Fig. 8 shows the repartition of energy along the x-direction 

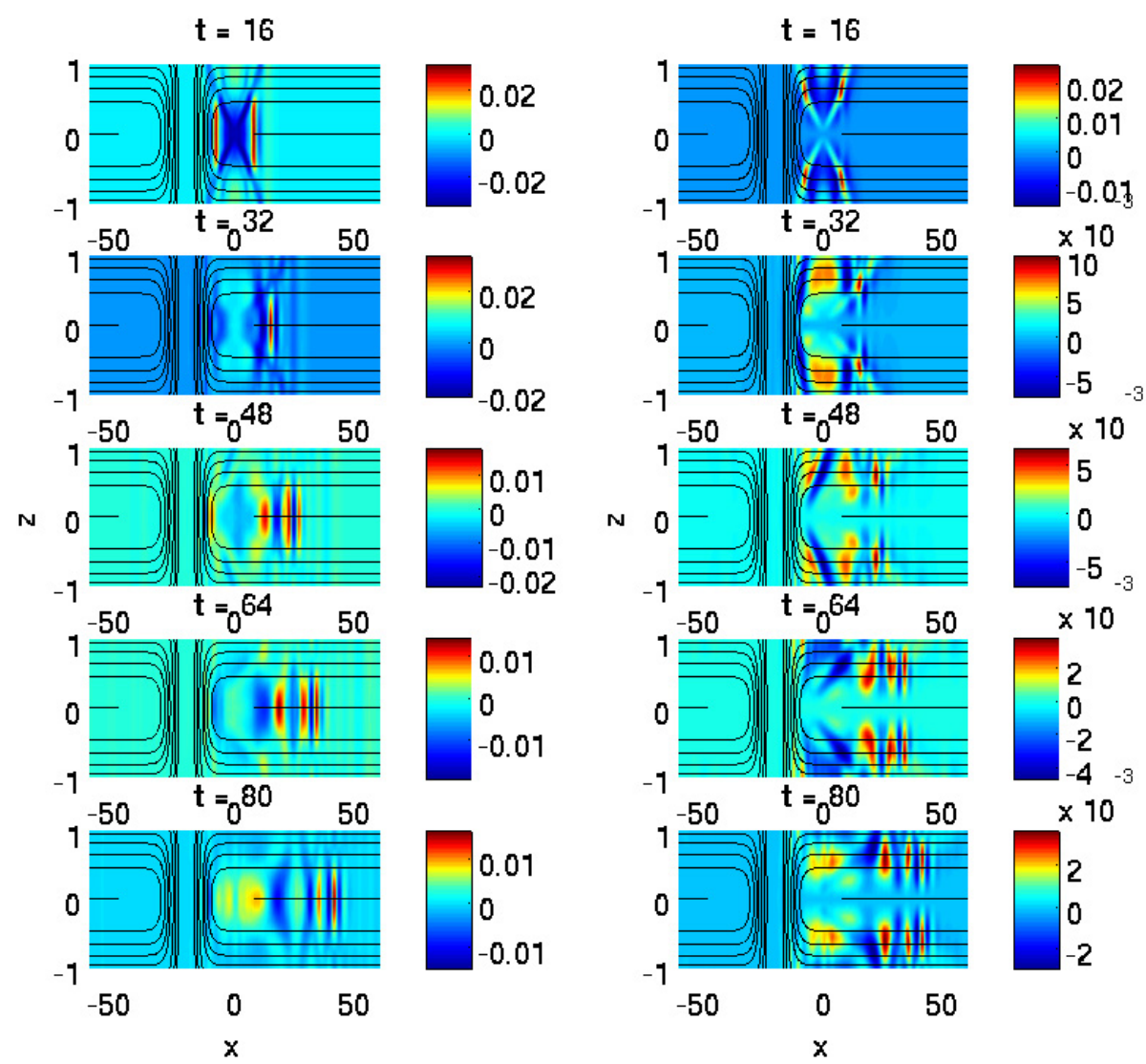

Fig. 9. Propagation of perturbations towards a magnetic X-point (with $\varepsilon=-0.8$ see text) located at $x=-20$ and $z=0$. Left column : thermal pressure fluctuations. Right column: magnetic pressure fluctuations. The pressure pulse explodes at the origin and sends two wave trains. The one propagating to the right remains unaffected but the wave train propagating to the X-point is blocked and reflected by the singularity. No fluctuations are observed on the other side of the X-point. Note that the X-direction is much compressed compared with the z-direction.

in the magnetotail at $t=20$ for two values of $B_{\mathrm{Z}}$ (solid line $B_{\mathrm{Z}}=0.01$ and dashed line $B_{\mathrm{Z}}=0.1$ ). The initial wave front carrying most of the energy is located at $x= \pm 18$ but the region in between where eigenmodes are expected to play an energetic role is almost empty in energy. The lower panel is a zoom of the upper panel in the range $-10<x<10$ and it can be seen that the energy density there represents only a few percent of the energy density transported by the sound wave. MHD eigenmodes seem to propagate efficiently only in a 1-D- or very close to a 1-D-configuration of the magnetotail. The presence of a significant normal component (above $0.1 B_{\text {ref }}$ ) destroys the structure of the eigenmodes. With an initial excitation modeled by a sudden gaussian pressure burst, a pure sound wave is generated and propagate the energy almost uniformly throughout the tail.

These simulations show that a finite $B_{\mathrm{Z}}$ component, even small, significantly changes the mode propagation in the plasma sheet and in some sense, the Harris sheet cannot be considered as a limiting case of a general 2-D-current sheet when $B_{\mathrm{Z}}$ tends to nought. To interpret this difference in behavior, one may notice that the MHD slow mode does not propagate energy across the magnetic field (the group velocity is zero) whereas the fast mode propagates energy almost isotropically. Strictly speaking, these modes should be considered only in uniform plasmas which is not the case here, but they provide a qualitative argument for MHD propagation. According to Fruit et al. (2002) eigenmodes propagate at a group velocity close to the speed of sound. They may thus be compared to slow modes. In a pure Harris sheet the energy carried by the eigenmodes cannot propagate in the z-direction, perpendicular to $\boldsymbol{B}_{0}$. It can flow only in the xdirection and stays confined within the plasma sheet if the driver lies on or near the x-axis. On the other hand, as soon as an even small $B_{\mathrm{Z}}$ is present in the structure, the energy carried by these slow modes is allowed to travel in the $\mathrm{z}$ direction as well as in the $\mathrm{x}$-direction along the magnetic field lines. The energy is then lost for the central plasma sheet and propagates mainly through this pure sound wave. 

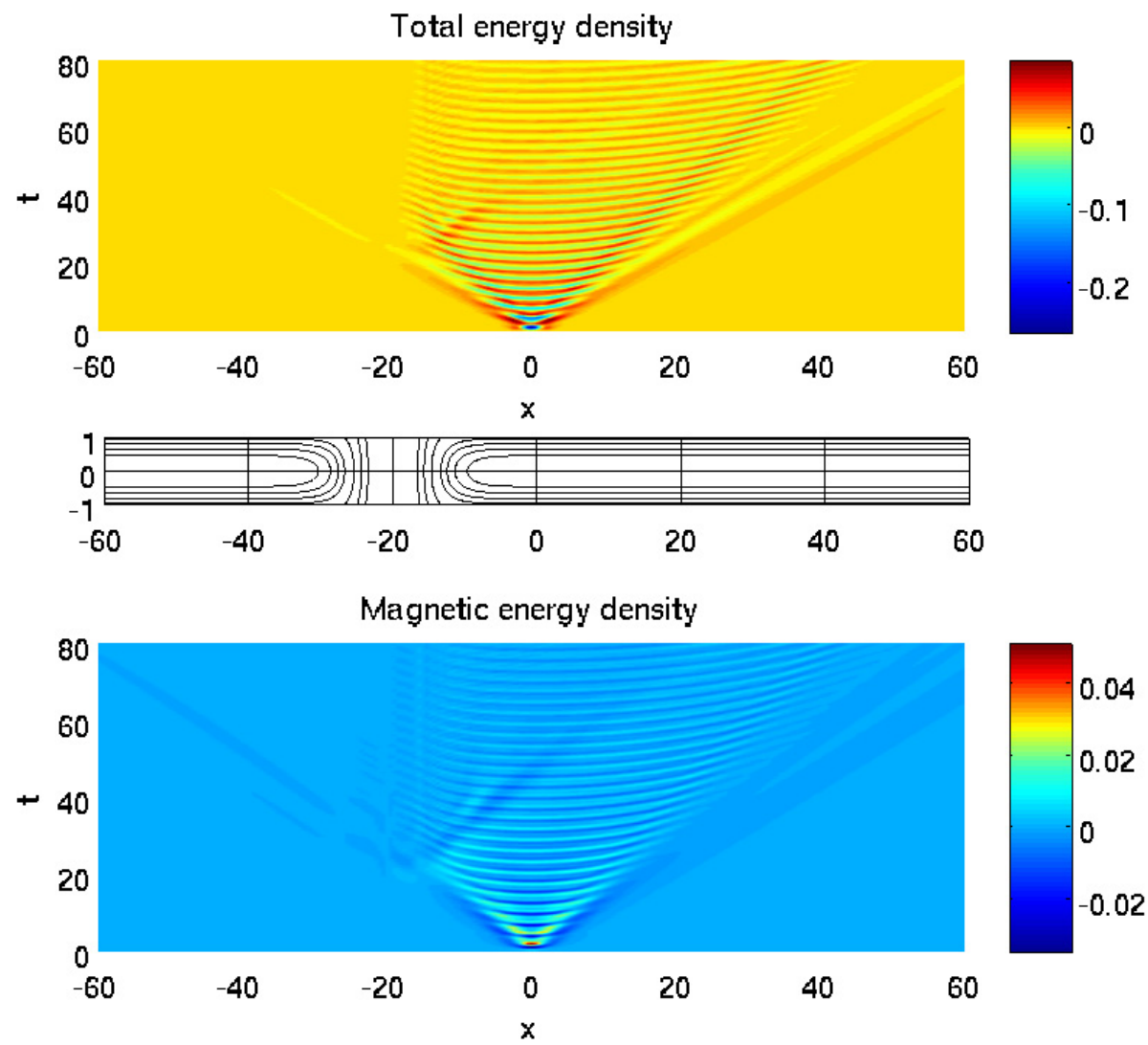

Fig. 10. Top panel: total magnetic energy of a vertical slice (integrated in the z-direction) $E_{t}(x, t)$ plotted versus $x$ and $t$ for the run showed in Fig. 9. Bottom panel: same for the magnetic energy density only. As showed in the central panel the X-point is located at $x=-20$. It is clearly visible that the energy does not propagate across the X-point and is even reflected towards the positive $x$.

\section{Propagation of MHD waves around null points}

An interesting application of the above study is to consider a current sheet containing a magnetic null point such as a $\mathrm{O}$ - or a X-point. In this configuration the $B_{\mathrm{Z}}$ component vanishes at the singular point but rises generally to a few $\mathrm{nT}$ in magnitude around the $\mathrm{X}$ - or $\mathrm{O}$-point. If this magnetic signature is strong enough it can become an obstacle to the propagation of MHD fluctuations along the sheet. Tur et al. (2001) proposes analytic solutions of Vlasov equation describing isolated X or O-points embedded into a Harris sheet. The magnetic flux function is given by

$\Psi_{0}(x, z)=-\ln \left(\frac{c(x)}{\cosh (c(x) z)}\right)$

with

$c(x)=1+\varepsilon \exp \left(\frac{x^{2}}{2 L_{\mathrm{x}}^{2}}\right)$

is the local half thickness of the Harris sheet. If $\varepsilon=0$ the pure 1-D-Harris sheet is recovered. If $\varepsilon>0$ (25) describes an O-point centered at the origin with typical size $L_{\mathrm{x}}$ along the plasmasheet. If $\varepsilon<0$ the singular point is an $\mathrm{X}$-point. The advantage of this solution lies in its rigorous derivation from the Grad-Shafranov equation and it can thus be applied in either MHD or kinetic models. Unfortunately it is only valid for $|z| \lesssim 1$ and becomes non realistic for $|z|>1$. This is not a severe drawback however, because we are mainly interested in the perturbations propagating within the current sheet, so we can concentrate on the zone $|z|<1$ and impose an artificial dissipation mechanism for $|z|>1.5$ which absorbs the energy flowing outwards of the sheet. In the following runs we illustrate the influence of an X-point placed at $x=-20$ on the propagation of the perturbations initiated by the pressure burst (2) still blasting at the origin. The shape of the X-point is chosen to exhibit a relatively strong $B_{\mathrm{Z}}$ in the left half of the plasma sheet, the right half remaining almost a pure 1-D Harris sheet. For $\varepsilon=-0.8$ and $L_{\mathrm{X}}=5$, it can be showed that the $B_{\mathrm{Z}}$ component reaches a maximum of $0.25 B_{\text {ref }} \simeq 6 \mathrm{nT}$.

Figure 9 proposes the result of a run: the left column represents the thermal pressure fluctuations and the right column the magnetic pressure fluctuations as contour plots in the (x,z)-plane for three values of time $t=16,32,48,64$ and 80. While the pressure eigenmodes develop and propagate freely towards the positive $\mathrm{x}$-direction, they are blocked, compressed and reflected by the presence of the singular 


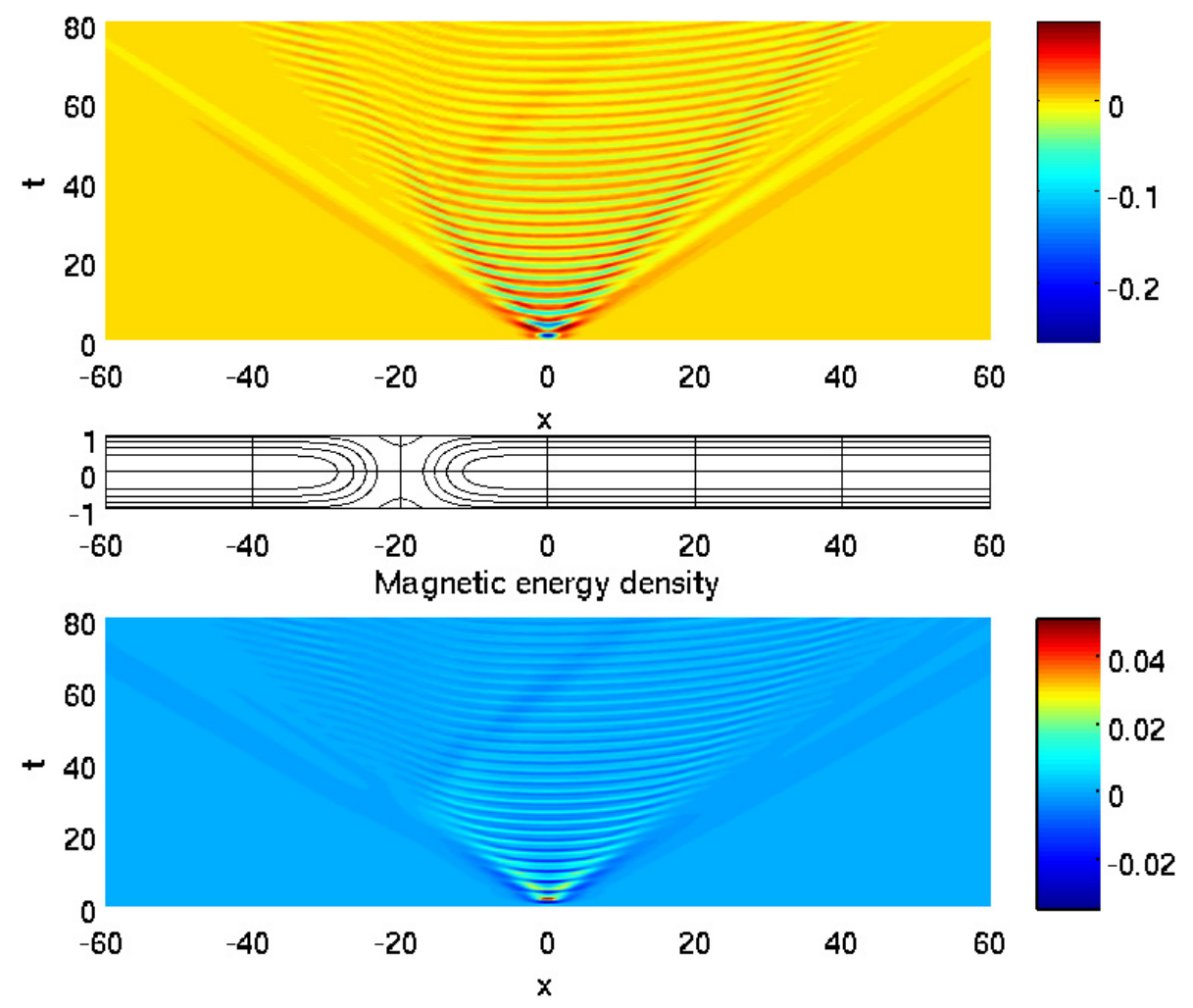

Fig. 11. Same figure as Fig. 10 but with a weaker $X$-point $(\varepsilon=-0.4)$. The $X$ point is still located at $x=-20$. The thermal fluctuations are able to cross or to bypass the X-point and after 40 Alfvén time thermal energy is observed for $x<-20$, but magnetic energy remains blocked by the singularity.

X-point. No magnetic or pressure fluctuations may be observed on the other side of the X-point which plays the role of an obstacle to the propagation of energy in the current sheet. To confirm this point the Fig. 10 shows the total (magnetic) energy stored in a vertical slice between $z= \pm 1$ and located at a given $x$ for a given time $t$. The propagation of energy at a constant group velocity towards the positive direction is obvious. The maximum group velocity may be evaluated at $0.5 v_{\mathrm{A}}$. The energy of the initial burst also spreads towards the negative $\mathrm{x}$-direction at the same group velocity as long as the $B_{\mathrm{Z}}$ component is weak. While approaching the coordinate $x \simeq-18$ where $B_{\mathrm{Z}}$ is highest, most of the energy is reflected towards the right, some is probably lost towards the edge of the current sheet $(z>1)$ but no energy is able to cross the singular point.

This result should however be tempered by the fact that the X-point geometry considered here is quite strong and does not necessarily represent the reality. Figure 11 shows the propagation of energy density in a weaker case with $\varepsilon=-0.4$. The maximum $B_{\mathrm{z}}$ along the neutral sheet is now $0.05 B_{\text {ref }} \simeq 1.5 \mathrm{nT}$. Here the same transition is observed at $x=-18$ but some energy density may flow across the Xpoint in that case. But the magnetic energy density remains quite low in the $x<-20$ region showing that only the thermal fluctuations are able to pass trough the singularity. Magnetic perturbations remains stopped by the magnetic barrier.

\section{Summary and conclusion}

The present paper aims to investigate the influence of a finite $B_{\mathrm{Z}}$ on the propagation of MHD perturbations. We solve an initial value problem where perturbations are excited by a sudden force field which may correspond to a pressure burst or a current disruption. The standard linear ideal MHD equations are then solved to compute the evolution of the thermal pressure and magnetic oscillations. As this system of partial differential equations is now 2-D, no analytic method may be used and a numerical solution is proposed here. First the 1-D-case is examined in order to recover the classical kink and sausage eigenmodes which compose the linear MHD response of the plasma sheet. The numerical solution shows also that the eigenmode structure is preceded by a fast wave front which propagates isotropically at the local Alfvén velocity. This fast wave takes about half of the initial energy of the pulse and spreads it in the global magnetotail. If this fast wave has a non vanishing $k_{\mathrm{y}}$ component (which is not considered here), it may excite resonantly a shear Alfvén wave propagating along the line of force and driving field aligned currents. Here, as $k_{\mathrm{y}}=0$ such phenomenon does not occur and the fast wave propagates freely. The eigenmodes propagating within the plasma sheet at a velocity close to $v_{\mathrm{s}}$ keeps the other half of the initial energy. 
The introduction of a finite $B_{\mathrm{Z}}$ changes these features quite deeply. While an eigenmode structure still develops with moderate $B_{\mathrm{Z}}$ (less than $0.01 B_{\text {lobes }}$ ), it propagates at a much lower speed along the $\mathrm{x}$-axis. The linear response of the plasma sheet is still composed of two wave trains: a first wave front identified as a sound wave propagates isotropically at $v_{\mathrm{s}}$ from the initial burst. An eigenmode structure develops next but it propagates at a much lower speed along the $\mathrm{x}$-axis and contains relatively little energy. About $99 \%$ of the initial energy of the burst is carried away by the sound wave and only a few percents are left within the plasma sheet. This dissymmetry in the distribution of the energy is enhanced with the value of $B_{\mathrm{z}}$. The MHD eigenmodes require that the magnetotail adopts an almost 1-D geometry to propagate along the field lines.

As a consequence of the above result, the propagation of MHD waves around a singular null point is also considered. Such a singularity embedded in a Harris sheet is surrounded by a finite $B_{\mathrm{Z}}$ which becomes an obstacle for the propagation of MHD eigenmodes. The numerical simulation of magnetic fluctuations propagating towards a magnetic $\mathrm{X}$-point surrounded by a $B_{\mathrm{Z}}$ reaching 5-6nT shows that only thermal fluctuations can cross the singularity, the magnetic energy is reflected backwards. If the singularity is weaker with $B_{\mathrm{Z}} \sim 1-2 \mathrm{nT}$, some magnetic oscillations are observed on the other side of the X-point which means that the reflection of magnetic energy is not total, but close to $90 \%$. Hence, an isolated singularity embedded in the plasma sheet seems to behave as a magnetic barrier for low frequency fluctuations. If several singular points are present, for instance as a result of a tearing instability, the low frequency fluctuations will stay trapped in between two X-points and new normal modes could thus appear due to this particular configuration.

Acknowledgements. Topical Editor I. A. Daglis thanks S. Fujita and another anonymous referee for their help in evaluating this paper.

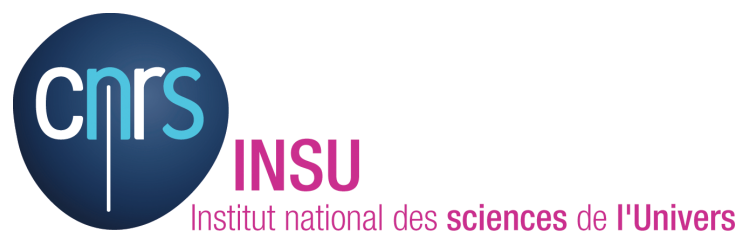

The publication of this article is financed by CNRS-INSU.

\section{References}

Allan, W. and Wright, A. N.: Magnetotail waveguide: Fast and Alfvén waves in the plasma sheet boundary layer and lobe, J. Geophys. Res., 105, 317-328, doi:10.1029/1999JA900425, 2000 .
Chen, L. and Hasegawa, A.: Plasma heating by spatial resonance of Alfven wave, Phys. Fluids, 17, 1399-1403, 1974.

Erkaev, N. V., Semenov, V. S., Kubyshkin, I. V., Kubyshkina, M. V., and Biernat, H. K.: MHD model of the flapping motions in the magnetotail current sheet, J. Geophys. Res. (Space Physics), 114, 3206, doi:10.1029/2008JA013728, 2009a.

Erkaev, N. V., Semenov, V. S., Kubyshkin, I. V., Kubyshkina, M. V., and Biernat, H. K.: MHD aspect of current sheet oscillations related to magnetic field gradients, Ann. Geophys., 27, 417-425, doi:10.5194/angeo-27-417-2009, 2009b.

Fruit, G., Louarn, P., Tur, A., and LeQuéau, D.: On the propagation of magnetohydrodynamic perturbations in a Harris-type current sheet: 1. Propagation on discrete modes and signal reconstruction, J. Geophys. Res., 107, 1411, doi:10.1029/2001JA009212, 2002.

Fruit, G., Louarn, P., Budnik, E., Sauvaud, J. A., Jacquey, C., Le Quéau, D., Rème, H., Lucek, E., Balogh, A., and CornilleauWehrlin, N.: On the propagation of low-frequency fluctuations in the plasma sheet: 2. Characterization of the MHD eigenmodes and physical implications, J. Geophys. Res. (Space Physics), 109, 3217, doi:10.1029/2003JA010229, 2004.

Lee, K. and Hau, L.: Characteristics of magnetohydrodynamic waves in Harris-type current sheet with guide magnetic field $B_{y}$, J. Geophys. Res. (Space Physics), 113, 12209, doi:10.1029/ 2008JA013459, 2008.

Lembege, B. and Pellat, R.: Stability of a thick two-dimensional quasineutral sheet, Phys. Fluids, 52, 1995-2004, 1982.

Louarn, P., Fruit, G., Budnik, E., Sauvaud, J. A., Jacquey, C., Le Quéau, D., Rème, H., Lucek, E., and Balogh, A.: On the propagation of low-frequency fluctuations in the plasma sheet: 1. Cluster observations and magnetohydrodynamic analysis, J. Geophys. Res. (Space Physics), 109, 3216, doi:10.1029/ 2003JA010228, 2004.

Lysak, R. L., Song, Y., and Jones, T. W.: Propagation of Alfvén waves in the magnetotail during substorms, Ann. Geophys., 27, 2237-2246, doi:10.5194/angeo-27-2237-2009, 2009.

Seboldt, W.: Nonlocal analysis of low-frequency waves in the plasma sheet, J. Geophys. Res., 95, 10471-10479, 1990.

Sedlacek, Z.: Electrostatic oscillations in cold inhomogneneous plasma. Part 1: differential equation approach, J. Plasma Physics, 5, 239-263, 1971.

Smith, J. M., Roberts, B., and Oliver, R.: Magnetoacoustic wave propagation in current sheets., Astrophys. and Astrom., 327, 377-387, 1997.

Tamao, T.: Transmission and coupling resonance of hydromagnetic disturbances in the non-uniform Earth's magnetosphere, Sci. Rep. Tohoku Univ., Series 5, Geophysics 17, 1965.

Tirry, W. J., Cadez, V. M., and Goossens, M.: MHD surface type quasi-modes of a current sheet model, Astron. Astrophys., 324, 1170-1178, 1997.

Tur, A., Louarn, P., Yanovsky, V., Le Queau, D., and Genot, V.: On the asymptotic theory of localized structures in a thin two-dimensional Harris current sheet: plasmoids, multiplasmoids and X points, J. Plasma Phys., 66, 97-117, doi:10.1017/ S002237780100112X, 2001. 